\title{
The Reamer-Irrigator-Aspirator: Roles and Evidence Supporting its use in Current Orthopaedic Practice
}

Kevin M. Kuhn*, Brian T. Barlow and David Dromsky

Department of Orthopaedic Surgery, Naval Medical Center San Diego, USA

\begin{abstract}
The Reamer-Irrigator-Aspirator (RIA) is an innovative technology created to address physiologic consequences of intramedullary reaming in femur fractures. While multiple physiolgic benefits have been demonstrated in animal studies, currently, no high quality data to demonstrate improved clinical outcomes in trauma patients or support its routine use in the treatment of acute femur fractures. Auto graft obtained from the use of RIA demonstrates osteogenic potential at least equal to iliac crest auto graft with potentially less donor site morbidity. The RIA also demonstrates theoretical benefits in the treatment of conditions which require intramedullary debridement; however, at this time no high level comparative trials exist to support its routine use. Although new complications unique to the RIA device have been reported, most can be avoided with careful preoperative planning and meticulous attention to technique. The RIA has many promising characteristics, but further research is necessary to continue to clarify its clinical role in modern orthopaedics.
\end{abstract}

Keywords: Reamer-irrigator-aspirator; RIA; Modern orthopaedics

\section{Introduction}

Gerhard Kuntscher [1] first recognized that placing larger intramedullary devices enhanced the healing of long bone fractures. By the mid-1950's he had adopted motorized reaming as a standard part of his technique. However, he also recognized that intramedullary fat could be a source of potentially fatal embolus if introduced systemically. To avoid this complication, he recommended over sizing the entry portal to allow the intramedullary contents to escape during insertion, as well as delaying intramedullary instrumentation in hemodynamically unstable patients [1].

The general understanding of the physiologic effects of intramedullary reaming improved over the next 50 years. Thermal and pulmonary effects were studied and investigators developed crude irrigation-suction systems to reduce heat generation, reduce intramedullary pressure, and minimize embolization of intramedullary contents [2,3]. By the late 1990's the RIA system had been designed and patented and after several refinements, it was made available for clinical use by 2003. The Reamer-Irrigator-Aspirator (RIA) system was originally designed to provide all the benefits of intramedullary reaming while reducing potentially harmful physiologic effects. However, as often happens with new technology, its indications expanded as it became more widely used. As the osteogenic potential of the collected reamings was quantified, the device and technique began to be used as a source of auto graft harvest for treatment of bone defects, nonunions and in arthrodesis procedures. In addition the unique features of the device have been applied to debridement of the intramedullary canal in cases of long bone osteomyelitis, retained intramedullary cement and treatment of metastatic bony lesions. Also, accompanying any new medical technology is a new set of unique complications associated with the device whichhave been described. The RIA is now readily available in the United States and commonly used by many surgeons who routinely treat orthopedic trauma.

\section{Acute Care of Femur Fractures}

Femoral intramedullary nailing is the standard of care for femoral shaft fractures in the skeletally mature, but is not without associated risks. Intramedullary reaming temporarily reduces cortical perfusion by over $80 \%$, generates significant heat, and is known to embolize marrow contents with subsequent systemic complications, particularly on the pulmonary system $[2,4,5]$. Pulmonary dysfunction has multiple causes, including mechanical occlusion of the pulmonary vasculature, changes in the pulmonary artery pressure, and activation of the coagulation and immune systems by intravascular fat. The overall physiologic condition of the patient determines whether these effects will be clinically significant.

Early research on reaming showed that intramedullary pressure could be reduced with narrow, flexible reamer shafts, a long head taper, and enlarged sharp cutting flutes. Researchers later became interested in suction and venting of the femoral canal to potentially reduce the thermal and embolic effects of reaming. When 2 groups of intact sheep femurs were reamed, one with a Rinsing Suction Reamer (RSR) and the other with a conventional reamer, the RSR group showed no increase in intramedullary pressure over normal physiologic values, decreased fat intravasation into the pulmonary circulation, and improved cardiac and pulmonary hemodynamics [6]. In pigs reamed with the RIA there is evidence of improved cardiopulmonary function and fewer pulmonary emboli [7]. A subsequent study by the same group found decreased pulmonary activation of coagulation and fibrinolysis in the RIA group [8]. In a sheep pulmonary contusion model, animals reamed with the RIA had decreased pulmonary permeability and edema, decreased pulmonary leukocyte diapedesis, and less activation of the coagulation cascade, leading researchers to conclude that the systemic effects of reaming can be minimized by reamer design [9]. Finally, in a porcine hemorrhagic shock model, investigators demonstrated significant improvements in mean arterial pressure, pulmonary arterial pressure, arterial partial pressure of oxygen, and cardiac outputin the RIA treated groupand fewer marrow emboli in the pulmonary circulation and parenchyma compared to the non-RIA treated pigs [10]. Animal data confirms that the RIA decreases intramedullary

*Corresponding author: Kevin M. Kuhn, Department of Orthopaedic Surgery Naval Medical Center San Diego, San Diego, CA 92134, USA, E-mail: kevin.kuhn@med.navy.mil

Received December 09, 2013; Accepted January 28, 2014; Published February 05, 2014

Citation: Kuhn KM, Barlow BT, Dromsky D (2014) The Reamer-Irrigator-Aspirator: Roles and Evidence Supporting its use in Current Orthopaedic Practice. Surgery Curr Res 4: 167. doi:10.4172/2161-1076.1000167

Copyright: $\odot 2014$ Kuhn KM, et al. This is an open-access article distributed under the terms of the Creative Commons Attribution License, which permits unrestricted use, distribution, and reproduction in any medium, provided the original author and source are credited. 
pressure, temperature, and fat embolization, and attenuates many of the pathologic processes seen in the setting of pre-existing lung contusion or hypovolemia. However there are currently no studies in humans to show improved clinical parameters when the RIA is used. A pair of human trials showed decreased IL- 8 and IL 10 levels, and reduced amounts of embolized fat presented to the right atrium in patient with femur fractures treated with RIA reaming $[3,11]$. However, the authors did not report on clinical outcomes.

The sole study comparing clinical outcomes in trauma patients is a retrospective review of 156 patients treated for diaphyseal femur fractures with reamed intramedullary nails using either RIA or conventional reaming. The authors found no benefit of RIA compared to conventional reaming with respect to mortality, pulmonary complications, ICU stay, mechanical ventilation time, length of stay, or blood loss. The authors did however note a nonsignificant increase in fracture healing complications with RIA compared to conventional reaming [12].

At this time there is insufficient evidence to support the routine use of RIA in the treatment of acute femur fractures. However the physiologic benefits of this technology seem promising and warrant further study.

\section{Autogenous Bone Graft Harvest}

Kuntscher [1] recognized the osteogenic potential of intramedullary reaming products, and investigators realized the RIA system had potential as a bone graft harvester early in its development. Since 1986 we have known that traditional reaming products contain viable bone, osteocytes, and mesenchymal stem cells. Investigators began to specifically study RIA reaming products as bone graft, demonstrating both osteoinductive and osteoconductive properties, and the ability to form mechanically viable callous [13]. Multiple studies revealed that RIA bone graft contains growth factors and stem cell concentrations that either match or exceed those found in iliac crest bone $[14,15]$. Surgeons used bone collected fromthe RIA device for graftingand presented small retrospective studies along with descriptive technique articles that demonstrated excellent clinical efficacy in nonunions, segmental defects, and arthrodeses [16,17].

While iliac crest bone grafting is the current gold standard for auto graft harvest, it has the disadvantages of limited volume and historic donor site morbidity. The RIA system offers the advantageof large volume harvest $\left(30-90 \mathrm{~cm}^{3}\right)$ from accessible locations using a technique familiar to many orthopaedic surgeons with minimal patient risk.

RIA is a reliable technique for bone grafting procedures requiring greater than $20 \mathrm{cc}$ of auto graft. RIA auto graft harvest is commonly performed on the femur. Using the RIA to harvest tibial auto graft is possible butresults in a smaller volume compared to the femur. As with intramedullary nailing femur fractures, donor site sequelae can be expected around the hip for ante grade technique and about the knee for retrograde technique. For larger volume defects, an antegrade technique is preferred as the guide wire can be bent to direct the RIA into the femoral condyles for additional graft. A retrograde technique can be considered for midsize defects as positioning and starting point access may be easier. After retrograde harvest, the medullary canal is an open conduit for continued blood loss into knee and can result in large hemarthrosis. A useful technique recently reported uses an allograft bone dowel to seal the femoral canal to prevent ongoing bleeding [18] (Figure 1).

Both iliac crest graft and RIA graft are cancellous grafts that lack structural properties. Compared to iliac crest graft, the autologous graft

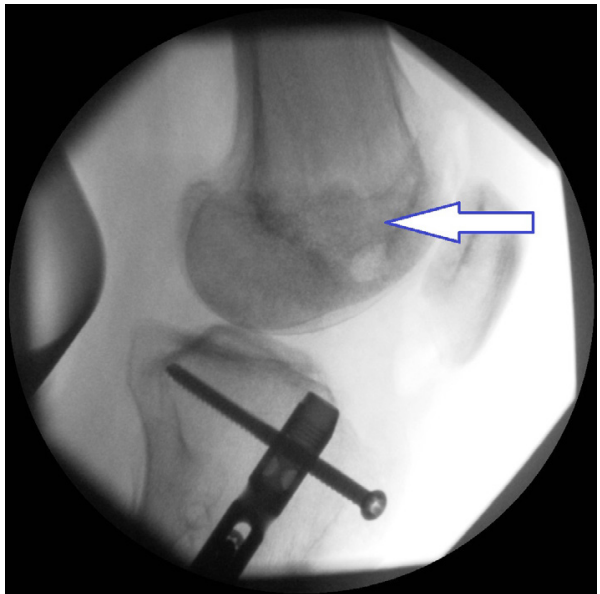

Figure 1: Intraoperative lateral fluoroscopic image of the knee following retrograde RIA harvest in a patient with a segmental tibial defect requiring bone grafting. The arrow demonstrates the allograft bone plug placed in the entry portal after harvest to prevent postoperative hemarthrosis.

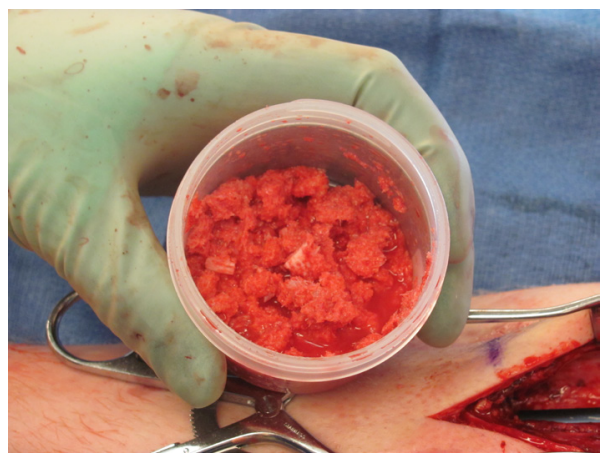

Figure 2: Intraoperative photograph of RIA harvest after being mixed with $15 \mathrm{cc}$ of allograft chips. The allograft acts as an expander and also helps to improve the handling characteristics of the RIA graft which is helpful when using in uncontained defects.

obtained using RIA has is more of slurry. Both types of cancellous graft can be mixed with allograft cancellous chips to expand the volume if needed (Figure 2).

\section{Treatment of Osteomyelitis, Tumors and Intramedullary Debris}

Thorough debridement of the intramedullary canal is an essential component of the treatment of long bone osteomyelitis. The RIA device has been used in small numbers to augment the treatment of intramedullary osteomyelitis [19-21]. The design RIA has several theoretical advantages that may make it a more preferred technique than conventional reaming. First, continuous irrigation and suction assists with the complete removal of infected bone debris, andthe collected debris can then be sent for microbiological analysis (Figures $3 \mathrm{a}$ and $3 \mathrm{~b}$ ). Second, continuous irrigation and sharp cutting flutes limit heat generation and, therefore, endosteal thermal necrosis. Third, continuous irrigation and suction decreases intramedullary pressure which may limit the bioburden and dissemination of the bacteria [22]. Septic emboli created during conventional reaming can disseminate throughout the body and theoretically cause infection elsewhere [21], although it is unclear whether this is clinically relevant. Finally, the potential benefits using RIA to perform intramedullary debridement 


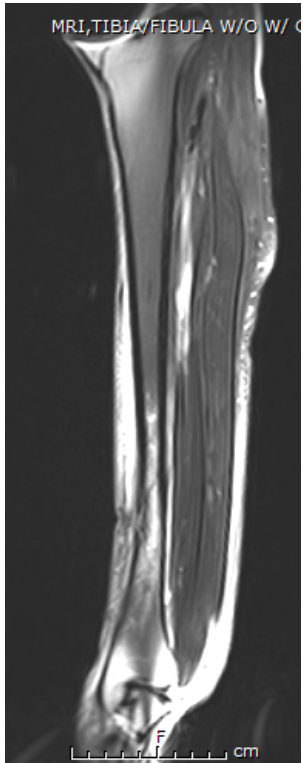

Figure 3a: Coronal MRI image of a male patient who developed increasing pain and adraining sinus 3 months after a gunshot wound to his leg which grazed the anteriorcortex of his tibia. The image demonstrates increased signal in the intramedullary canaland the sinus tract consistent with osteomyelitis. He was treated with a single stage open debridement and reaming of his canal with the RIA.

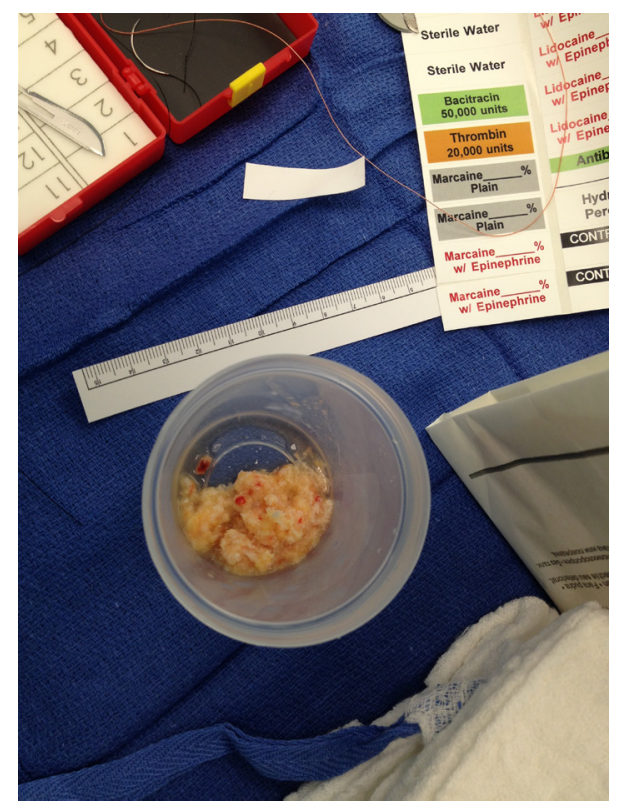

Figure 3b: Clinical photo of specimen cup with the collections from the RIA after reamingthe canal. The intramedullary contents were sent to the lab for microbiological analysis. The isolated organism was then used to direct antibiotic treatment.

for treating osteomyelitis in a physiologically compromised patient may result in less pulmonary embolic load during the procedure and diminish the systemic burden of the procedure.

There are no comparisons of conventional reaming versus RIA for the treatment of intramedullary osteomyelitis in the literature. Comparison of the irrigant volume with the RIA versus conventional reaming has not been reported in the current literature.The RIA system is a significant added operative expense and whether the RIA device provides superior outcomes in the treatment of intramedullary osteomyelitis has not been demonstrated in the current literature.

Other potential uses of the RIA device include the removal of retained intramedullary cement and removal of metastatic tissue in oncology cases. The RIA has also been reported to be effective in the removal of retained intramedullary cement [23]. Using RIA to ream long bones with metastatic lesions in an attempt to prevent dissemination of tumor cells has also been reported [21]. While evidence is limited, the unique features of the RIA make it useful for debriding the intramedullary canal in a wide variety of cases.

\section{Complications}

The most commonly reported and most feared complication of RIA use is fracture of the long bone, especially following large volume autogenous bone graft harvest. Low energy fractures of the femur have been reported on multiple occasions after large volume harvest of bone graft using the RIA device [24,25]. Silva et al. [26] conducted a cadaveric biomechanical study that compared intact femurs to post-RIA femurs. There was no significant difference in torsional strength and load capacity between intact femurs and post-RIA femurs when reamed $<2 \mathrm{~mm}$ greater than the isthmic diameter. However, the authors found that eccentric reaming negatively affected the strength characteristics and predisposed to fracture. Overreaming the isthmic diameter by $>2$ $\mathrm{mm}$ is not recommended as this may change the torsional and load characteristics of the long bone.

Caution should be exercised when selecting RIA in osteoporotic patients. All patients should be counseled on the risk of fracture and consideration should be given to prophylactic nail placement after RIA in osteoporotic or unreliable patients. Patients should also be counseled that prophylactic stabilization may be necessary in the event of over reaming, anterior cortical perforation or eccentric reaming as these conditions predispose to early iatrogenic fracture.

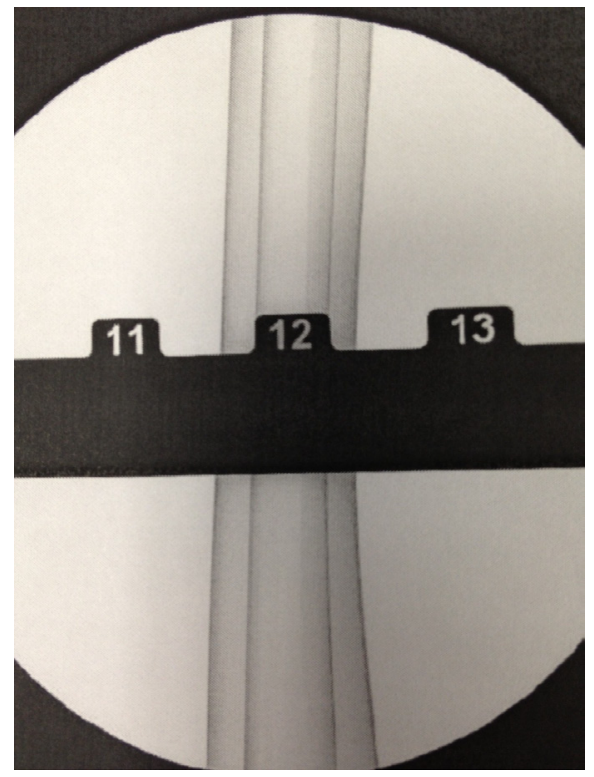

Figure 4: Representative drawing of the intraoperative technique used to select thereamer head size for the RIA. A radiolucent ruler is placed over the thigh to indentify the appropriate isthmal diameter. A reamer head 1.5 to 2.0 $\mathrm{mm}$ larger is then chosen. 
The sharp end-cutting flutes and the more rigid shaft of the RIA device may led to eccentric reaming with subsequent anterior femur cortical perforation, especially in patient with excessive femoral bow [25]. Frequent fluoroscopic imaging has been suggested as a way to prevent this complication. Careful preoperative imaging of the femur that is going to be harvested to assess the canal diameter and femoral bow is recommended. We recommend measuring the isthmus to confirm this measurement intraoperatively with a fluoroscopy and a radiolucent ruler held over the isthmus (Figure 4). A reamer head 1.5 to $2.0 \mathrm{~mm}$ larger than the isthmic diameter is then selected, although some authors advocate oversizing by as much as $3.0 \mathrm{~mm}$ larger [14]. The smallest reamer head available for the RIA is $12.0 \mathrm{~mm}$, so if preoperative imaging reveals as very narrow intramedullary canal one should seek an alternative source of bone graft.

There is some theoretical concern that use of the RIA in acute fracture may lead to increased rates of nonunion. Conventional reaming may increase healing of long bone fractures by providing an autogenous bone grafting at the fracture site or by inducing a healing response via increased periosteal blood flow secondary to the mechanical debridement of the medullary canal. The RIA device irrigates and suctions out medullary contents while reaming. The loss of the normal autogenous bone grafting effect of conventional reaming with irrigation and suction may contribute to the increased nonunion rate seen anecdotally with RIA use [12].

Excessive blood loss with resultant hypotension and tachycardia requiring transfusion has been reported with use of the RIA device. Irrigation and suction should be stopped when not actively reaming to limit blood loss and the surgeon should be vigilant about making the harvest expeditious. Anesthesia providers should be aware of possibility for excessive blood loss with the RIA procedure.

Similar to iliac crest harvest, other less common and theoretical complications associated with the RIA device include hypertrophic scars, hematoma, chronic pain, RIA head disassembly within the femur, and deep infection $[16,20]$. A systematic review of 12 studies comparing RIA to iliac crest bone graft demonstrated a complication rate in iliac crest bone graft (19.4\%) that was approximately three times that seen with RIA (6\%) [27]. Patients should be counseled that complications can occur with RIA bone graft harvest,but may beless common than those seen with conventional iliac crest bone graft harvest.

\section{Conflicts of Interest}

The authors report no actual or potential conflict of interest in relation to this article. The views expressed in this article are those of the authors and do not necessarily reflect the official policy or position of the US Department of the Navy, the US Department of Defense, or the US Government.

\section{References}

1. Kuntscher GB (1958) The Kuntscher method of intramedullary fixation. J Bone Joint Surg Am 40-40A: 17-26.

2. Volgas DA, Burch T, Stannard JP, Ellis T, Bilotta J, et al. (2010) Fat embolus in femur fractures: a comparison of two reaming systems. Injury 41 Suppl 2 : S90-93.

3. Hartsock LA, Barfield WR, Kokko KP (2014) Randomized prospective clinical trial comparing reamer irrigator aspirator (RIA) to standard reaming (SR) in both minimally injured and multiply injured patients with closed femoral shaft fractures treated with reamed intramedullary nailing (IMN). Injury 41Suppl 2: S94-98.

4. Pape HC, Auf'm'Kolk M, Paffrath T, Regel G, Sturm JA, et al. (1993) Primary intramedullary femur fixation in multiple trauma patients with associated lung contusion--a cause of posttraumatic ARDS? J Trauma 34: 540-547.
5. Roberts CS, Pape HC, Jones AL, Malkani AL, Rodriguez JL, et al. (2005) Damage control orthopaedics: evolving concepts in the treatment of patients who have sustained orthopaedic trauma. Instructional course lectures 54: 447462.

6. Joist A, Schult M, Ortmann C, Frerichmann U, Frebel T, et al. (2004) Rinsing-suction reamer attenuates intramedullary pressure increase and fat intravasation in a sheep model. J Trauma 57: 146-151.

7. Husebye EE, Lyberg T, Opdahl H, Laurvik H, Røise O (2010) Cardiopulmonary response to reamed intramedullary nailing of the femur comparing traditional reaming with a one-step reamer-irrigator-aspirator reaming system: an experimental study in pigs. J Trauma 69: E6-14.

8. Husebye EE, Opdahl H, Røise O, Aspelin T, Lyberg T (2011) Coagulation, fibrinolysis and cytokine responses to intramedullary nailing of the femur: an experimental study in pigs comparing traditional reaming and reaming with a one-step reamer-irrigator-aspirator system. Injury 42: 630-637.

9. Pape HC, Zelle BA, Hildebrand F, Giannoudis PV, Krettek C, et al. (2005) Reamed femoral nailing in sheep: does irrigation and aspiration of intramedullary contents alter the systemic response? J Bone Joint Surg Am 87: 2515-2522.

10. Wang RY, Li R, Zdero R, Bell D, Blankstein M, et al. (2012) The physiologic and pathologic effects of the reamer irrigator aspirator on fat embolism outcome: an animal study. J Orthop Trauma 26: e132-137.

11. Streubel PN, Desai P, Suk M (2010) Comparison of RIA and conventional reamed nailing for treatment of femur shaft fractures. Injury 41 Suppl 2: S51-56.

12. Hammer TO, Wieling R, Green JM, Südkamp NP, Schneider E, et al. (2007) Effect of re-implanted particles from intramedullary reaming on mechanical properties and callus formation. A laboratory study. J Bone Joint Surg $\mathrm{Br} 89$ : $1534-1538$.

13. Sagi HC, Young ML, Gerstenfeld L, Einhorn TA, Tornetta P (2012) Qualitative and quantitative differences between bone graft obtained from the medullary canal (with a Reamer/Irrigator/Aspirator) and the iliac crest of the same patient. J Bone Joint Surg Am 94: 2128-2135.

14. Schmidmaier G, Herrmann S, Green J, Weber T, Scharfenberger A, et al (2006) Quantitative assessment of growth factors in reaming aspirate, iliac crest, and platelet preparation. Bone 39: 1156-1163.

15. McCall TA, Brokaw DS, Jelen BA, Scheid DK, Scharfenberger AV, et al. (2010) Treatment of large segmental bone defects with reamer-irrigator-aspirator bone graft: technique and case series. OrthopClin North Am 41: 63-73.

16. Newman JT, Stahel PF, Smith WR, Resende GV, Hak DJ, et al. (2008) A new minimally invasive technique for large volume bone graft harvest for treatmen of fracture nonunions. Orthopedics 31: 257-261.

17. Barlow BT, Kuhn KM (2012) Technique and indications for the retrograde use of the

18. reamer-irrigator-aspirator. Current Orthopaedic Practice. Epub.

19. Finkemeier CG, Neiman R, Hallare D (2010) RIA: one community's experience. Orthop Clin North Am 41: 99-103.

20. Kanakaris NK, Morell D, Gudipati S, Britten S, Giannoudis PV (2011) Reaming Irrigator Aspirator system: early experience of its multipurpose use. Injury 42 Suppl 4: S28-34.

21. Zalavras CG, Sirkin M (2010) Treatment of long bone intramedullary infection using the RIA for removal of infected tissue: indications, method and clinical results. Injury 41 Suppl 2: S43-47.

22. Goplen G, Wilson JA, McAffrey M, Deluzio K, Leighton R (2010) A cadaver model evaluating femoral intramedullary reaming: a comparison between new reamer design (Pressure Sentinel) and a novel suction/irrigation reamer (RIA). Injury 41Suppl 2: S38-42

23. Lowe JA, Vosburg C, Murtha YM, Della Rocca GJ, Crist BD (2011) A new technique for removing intramedullary cement. J Orthop Trauma 25: 762-766.

24. Giori NJ, Beaupre GS (2011) Femoral fracture after harvesting of autologous bone graft using a reamer/irrigator/aspirator. J Orthop Trauma 25: e12-14.

25. Lowe JA, Della Rocca GJ, Murtha Y, Liporace FA, Stover MD, et al. (2010) Complications associated with negative pressure reaming for harvesting autologous bone graft: a case series. J Orthop Trauma 24: 46-52.

26. Silva JA, McCormick JJ, Reed MA, Morse AS, Heffernan MJ, et al. (2010) Biomechanical effects of harvesting bone graft with the Reamer/lrrigator/ Aspirator on the adult femur: a cadaver study. Injury 41 Suppl 2: S85-89. 
Citation: Kuhn KM, Barlow BT, Dromsky D (2014) The Reamer-Irrigator-Aspirator: Roles and Evidence Supporting its use in Current Orthopaedic Practice. Surgery Curr Res 4: 167. doi:10.4172/2161-1076.1000167

27. Quintero AJ, Tarkin IS, Pape HC (2010) Technical tricks when using the reamer irrigator aspirator technique for autologous bone graft harvesting. $\mathrm{J}$ Orthop Trauma 24: 42-45
28. Dimitriou R, Mataliotakis GI, Angoules AG, Kanakaris NK, Giannoudis PV (2011) Complications following autologous bone graft harvesting from the iliac crest and using the RIA: a systematic review. Injury 42 Suppl 2: S3-15. 